\title{
Nanotexture effect of the fiber surface on the sorption capacity of nonwoven fabrics
}

\author{
Yu. V. Rudyak ${ }^{1}$, F. A. Doronin ${ }^{1}$, G. O. Rytikov ${ }^{1,2}$, E. K. Filyugina ${ }^{3}$, V. G. Nazarov ${ }^{1}$ \\ ${ }^{1}$ Moscow Polytechnic University, 38 B. Semenovskya str., Moscow 107023, Russia \\ ${ }^{2}$ State University of Management, 99 Ryazansky pr., Moscow 109542, Russia \\ ${ }^{3}$ National Research University Higher School of Economics, 20, Myasnitskaya Street, Moscow 101000, Russia \\ rudyak@mail.ru, f.a.doronin@mospolytech.ru,gr-yandex@yandex.ru, \\ ekaterina.filyugina@mail.ru, 110505n@gmail.com
}

DOI 10.17586/2220-8054-2020-11-5-553-564

\begin{abstract}
An approach to structural and functional modelling of the oxyfluorinated nonwovens based composite materials has been developed. The structural component of the model is presented in the form of integral and differential characteristic functions of the planar heterogeneity. The correlation analysis methods revealed the existence of links between the latex sorption capacities and the nanostructural characteristics of the experimental samples' SEM-images. The obtained functional-structural model allows us to quantify the expected values of sorption capacities of the nonwoven fiber materials under the certain restrictions on the chemical compositions of the impregnating mixtures.
\end{abstract}

Keywords: oxyfluorination, surface modification, fiber, modification, sorbtion capacity.

Received: 4 September 2020

Revised: 15 September 2020

\section{Introduction}

Woven and nonwoven fabrics are well-known and widespread options for implementing materials based on polymer fibers [1-3]. Unlike fabrics consisting of regularly ordered forming threads, nonwoven fabrics have a chaotic and fundamentally multi-layered structure, because, otherwise, they do not provide the necessary level of mechanical strength of finished products for practical operation [4-8]. A significant influence of finish treatment on the physico-chemical and as a result, functional and operational properties (wettability, sorption capacity, permeability, radioparadise, etc.) nonwoven fabric [9-11] can be used for solving a wide range of tasks (provide heat and moisture insulation, filtration from different different-scale admixtures in water and fuel, emergency spill response of fuel and lubricants, etc.) [12-14]. At the same time, the optical characteristics of different-scale visualizations of nonwoven fabric surfaces can significantly change under the influence of various finishing methods, which makes it possible to identify correlations between the functional and morphological characteristics of fibrous materials based on the analysis of their macro -, micro-and nanoscale images [15-17]. The study of the relationships between the set of parameters of manufacturing modes, structural and functional characteristics of the original and modified samples allows one to predict and control the properties of products used in various natural conditions, made on the basis and/or using fibrous materials. The identification of correlations between the structural and functional characteristics of the corresponding experimental samples makes it possible to clarify the mechanisms by which changes in functional properties are provided by the control program for the production of new materials based on nonwoven fabrics.

\section{Experimental samples}

Test non-woven fabrics were made on the basis of two-component mixtures of polyester fibers of various linear densities (0.33 and 1.7 Tex) using the technology described in [18]. Thermomechanical processing of experimental samples was performed at a temperature of $130^{\circ} \mathrm{C}$ and the speed of broaching non-woven fabrics between the heated shafts $12 \mathrm{~m} / \mathrm{min}$. Chemical treatment of samples was carried out by oxychlorination with a gas mixture of $7.5 \% \mathrm{~F}_{2}+10 \% \mathrm{O}_{2}+82.5 \% \mathrm{He}$ with processing times of $0.5,1,1.5$ and 2 hours.

The latex-sorption capacity of the experimental samples was subjected to a direct gravimetric measurement (Table 1). The latter was chosen as a simulation model of liquid-phase functional additives applied to the surface and staining non-woven fabrics in volume during final pre-operational processing.

Latex treatment was performed in four different modes, hereafter referred to as L1, L2, L3 and L4. Modified cloths were impregnated with latex copolymer of vinylidene chloride and butadiene (DVCB-70, 70\% butadiene and $30 \%$ vinylidene chloride) with a rubber content of 26\% (GOST 9501-60 synthetic Latex DVCB-70, TU 38.303-0403-90) at a temperature of $22^{\circ} \mathrm{C}$. To regulate the degree of impregnation, water-diluted latex with a rubber content of 3.5 (L 1), 4.4 (L2), 6.0 (L 3) and $13 \%$ (L4) was used. Samples immersed in latex were rolled with a roller for 
TABLE 1. The latex-sorption capacity SC for oxyfluorinated bicomponent polyester fiber materials under various modes of gas-phase modification and liquid-phase processing

\begin{tabular}{|c|c|c|c|c|}
\hline \multirow{2}{*}{$\begin{array}{c}\text { Duration of } \\
\text { oxyfluorination, } \mathrm{h}\end{array}$} & \multicolumn{4}{|c|}{ The impregnation mode } \\
\cline { 2 - 5 } & L1 & L2 & L3 & L4 \\
\hline \hline 0.5 & $3.8 \pm 0.4$ & $4.1 \pm 0.4$ & $4.9 \pm 0.5$ & $5.1 \pm 0.5$ \\
\hline 1.0 & $3.6 \pm 0.4$ & $3.3 \pm 0.3$ & $4.5 \pm 0.5$ & $4.2 \pm 0.4$ \\
\hline 1.5 & $3.6 \pm 0.4$ & $2.9 \pm 0.3$ & $3.8 \pm 0.4$ & $4.7 \pm 0.5$ \\
\hline 2.0 & $4.4 \pm 0.4$ & $2.5 \pm 0.3$ & $3.7 \pm 0.4$ & $4.5 \pm 0.5$ \\
\hline
\end{tabular}

2 minutes. Latex-impregnated non-woven fabrics were passed through pressing rollers and, when blown with air at a temperature of $120^{\circ} \mathrm{C}$, dried until constant values of sample weights were reached.

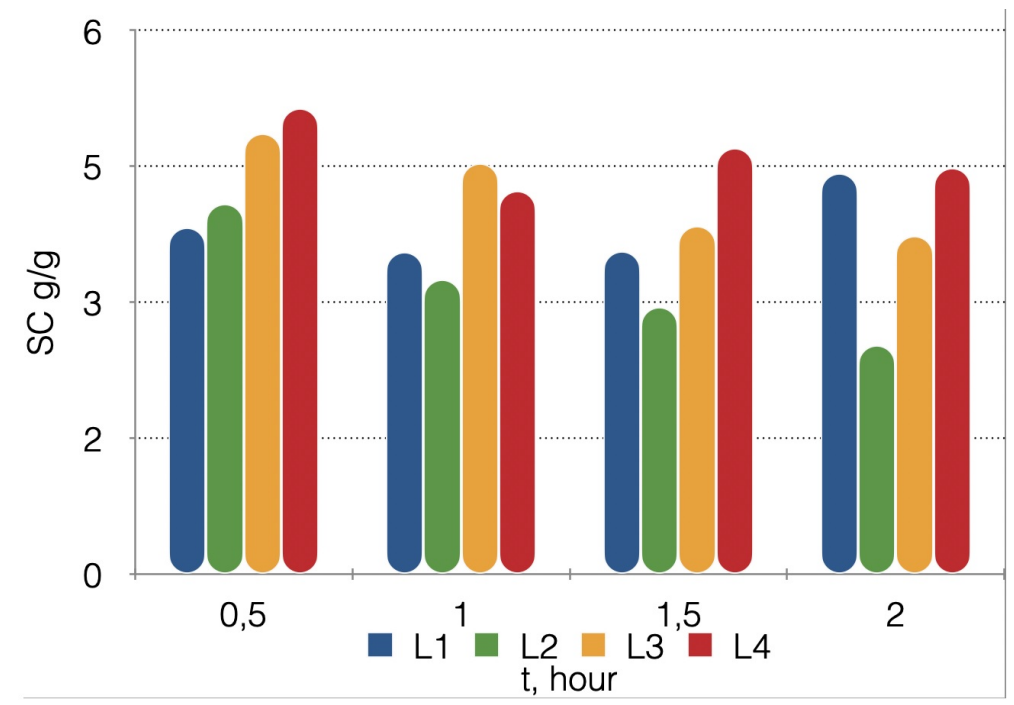

FIG. 1. Values of the SC sorption capacity for latex of experimental samples treated in L1, L2, L3 and L4 modes at different durations of oxyfluorination with a gas mixture of $7.5 \% \mathrm{~F}_{2}+10 \% \mathrm{O}_{2}+82.5 \% \mathrm{He}$

It is seen (Table 1, Fig. 1) that the different modes of impregnation with latex sorption capacity of samples in most cases decreases with increasing length $t$ of gas-phase chemical modification, but trends are not absolute in nature, which is probably related to the increase in the hydrophilicity of polyester fibers during the oxyfluorination and the dependence of sediment stabilities latex on the concentration of rubber in water.

\section{The average sorption capacity dependence on the duration of oxyfluorination}

Figure 2 shows the dependence of the average values of sorption capacities of experimental samples (regardless of the mode of latex impregnation) on the duration of oxyfluorination with a gas mixture of $7.5 \% \mathrm{~F}_{2}+10 \% \mathrm{O}_{2}+82.5 \%$ He for $0.5,1,1.5$ and 2 hours. The average value of the sorption capacity of the initial samples is represented by a point corresponding to the zero duration of gas-phase modification.

The dependence of the average value of the sorption capacity on the duration of oxyfluorination is well described by the equation:

$$
S C=S C_{0} \cdot e^{-t^{2}}+S C_{\infty}
$$

where $S C_{0}$ is the sorption capacity of the initial samples, $S C_{\infty}$ - is the conditional expected sorption capacity of samples oxyfluorinated over an infinitely long time, and $t$ is the dimensionless time.

The values of parameters of the functional-dynamic model (1) obtained by the generalized method of least squares (OLS), intended for predicting the average sorption capacity of bicomponent nonwovens when the duration of oxyfluorination changes, are presented in Table 2. 


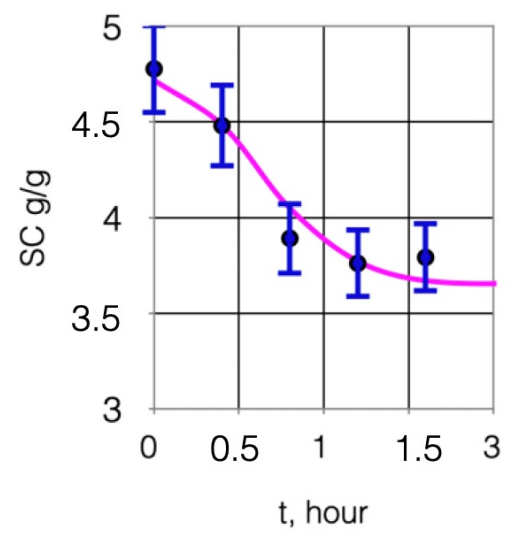

FIG. 2. Dependence of the average values of sorption capacities of experimental samples on the duration of oxyfluorination with a gas mixture of $7.5 \% \mathrm{~F}_{2}+10 \% \mathrm{O}_{2}+82.5 \% \mathrm{He}$ for $0.5,1,1.5$ and 2 hours

TABLE 2. Values of parameters of the functional-dynamic model obtained by the GLS (generalized least squares) method (1)

\begin{tabular}{|c|c|c|c|}
\hline Parameter & $S C_{0} \pm \delta S C_{0}, \mathrm{~g} / \mathrm{g}$ & $S C_{\infty} \pm \delta S C_{\infty}, \mathrm{g} / \mathrm{g}$ & $R^{2}$ \\
\hline Value & $1.1 \pm 0.1$ & $3.7 \pm 0.4$ & 0.95 \\
\hline
\end{tabular}

Thus, the dependence of the average sorption capacity of the sample on the duration of oxyfluorination shows the principal possibility of controlling the functional characteristics of composite materials based on non-woven fabrics using gas-phase chemical modification.

\section{The concept of structural model}

In order to ensure the efficiency of impregnation of non-woven fabrics, the highest for some and the lowest for other latex compositions (simulating various liquid-phase functional compounds applied), it is necessary to develop a technique for monitoring and predicting the properties of composite fiber materials based on the analysis of the corresponding optical images. For example, Fig. 3 shows enlarged optical front and profile images of one of the experimental samples after oxyfluorination.
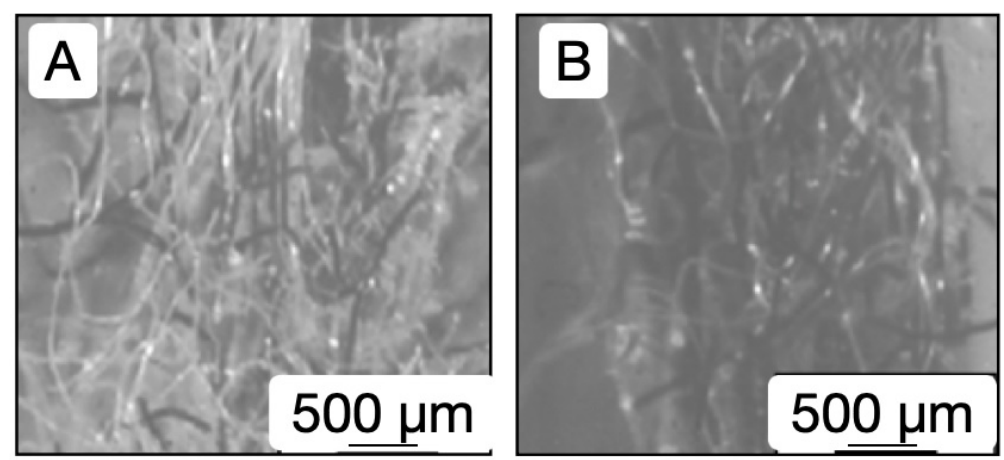

FIG. 3. Optical front (A) and profile (B) images of the experimental sample after oxyfluorination

A significant disadvantage of direct adaptation of the previously developed original approach to the formation of structural models in the form of decompositions into two-dimensional Fourier series is a large number of parameters, the specification of which is necessary to achieve high-quality approximation of digital images of optical images of analyzed objects. Mathematically strict verification of such models requires a large amount of experimental data, and its visualization involves the use of pseudo-three-dimensional images, which makes it difficult for researchers and engineers to perceive and qualitatively analyze the structural characteristics of the studied materials. 
To solve the above problem, an original approach to the quantitative description of images of experimental samples was developed, consisting in the formation of characteristic functions of planar digital heterogeneity, which formalize the dependence on the threshold values of pixel $B$ brightness of the normalized total area $S / S_{0}(B)$ of shapes formed by pixels of the analyzed images, the brightness of which does not exceed $B$. Each image of an experimental sample can be compared with an integral and differential characteristic function, determining the latter using a difference scheme $\Delta\left(S / S_{0}(B+1)-S / S_{0}(B)\right.$. The visual models of front-facing (A) and profile (B) images corresponding to different pixel brightness thresholds (Fig. 3) are shown in Table 3.

TABLE 3. Values of parameters of the functional-dynamic model for predicting the values of the average sorption capacity when changing the duration of oxyfluorination

\begin{tabular}{|c|c|c|c|c|c|c|c|c|}
\hline $\begin{array}{l}\text { Threshold } \\
\text { value } B \text { the } \\
\text { brightness of } \\
\text { pixels of the } \\
\text { images }\end{array}$ & 16 & 48 & 80 & 112 & 144 & 176 & 208 & 240 \\
\hline $\begin{array}{l}\text { Octile of image } \\
\text { pixel brightness } \\
\text { distribution }\end{array}$ & I & II & III & IY & $\mathrm{Y}$ & YI & YII & YIII \\
\hline $\begin{array}{l}\text { Visual } \\
\text { models } \\
\text { of the front } \\
\text { image }\end{array}$ & 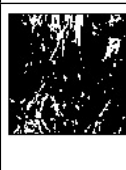 & $x$ & $\begin{array}{r}0 \\
-3 \\
-3\end{array}$ & $\ldots$ & 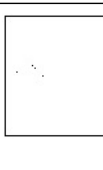 & & & \\
\hline$S /\left.S_{0}\right|_{\text {front }}$ & 0.16 & 0.69 & 0.89 & 0.98 & 0.997 & 0.9998 & 1 & 1 \\
\hline$\left.\Delta\left(S / S_{0}\right)\right|_{\text {front }}$ & 6.8 & 6.4 & 3.7 & 1.4 & 0.27 & 0.03 & 0 & 0 \\
\hline $\begin{array}{l}\text { Visual } \\
\text { models } \\
\text { of the profile } \\
\text { image }\end{array}$ & & & 4 & 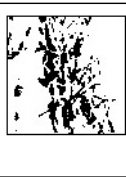 & 算 & is & 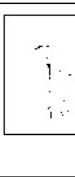 & \\
\hline$S /\left.S_{0}\right|_{\text {profile }}$ & $<0.01$ & 0.09 & 0.42 & 0.69 & 0.85 & 0.94 & 0.98 & $>0.99$ \\
\hline$\left.\Delta\left(S / S_{0}\right)\right|_{\text {profile }}$ & $<0.01$ & 2.7 & 6.6 & 7.5 & 5.5 & 3.4 & 1.4 & 0.3 \\
\hline
\end{tabular}

Thus, the functional dependences of the integral $S / S_{0}(B)$ and differential $\Delta S / S_{0}(B)$ functions of planar heterogeneity can be considered as unified quantitative structural characteristics of the samples under consideration.

The characteristic functions $S / S_{0}(B)$ and $\Delta S / S_{0}(B)$ describing the front and profile images of experimental samples oxyfluorinated with a mixture of $7.5 \% \mathrm{~F}_{2}+10 \% \mathrm{O}_{2}+82.5 \% \mathrm{He}$ for $0.5,1,1.5$ and 2 hours are shown in Fig. 4 and Fig. 5, respectively.

\section{Correlation and regression analysis of functional and structural relationships}

A quantitative assessment of the possibility of establishing in a certain sense "universal" (common to all optical images obtained under the same conditions of observation) threshold values $B$ of pixel brightness, at which the relationship between the values of characteristic functions $S / S_{0}(B)$ and $\Delta\left(S / S_{0}(B)\right)$ and the values of sorption capacities $(S C)$ of experimental samples is statistically significant, was performed using data correlation analysis methods.

The results of the correlation analysis (correlogram) of the structural and functional relationships of the sorption capacity with the parameters of the functions of integral and differential planar digital heterogeneity of frontal and profile images of experimental samples are shown in Fig. 6. The obtained values of Pearson correlation coefficients are stored on the ordinate axes; the threshold values of pixel brightness are stored on the abscissa axes.

It can be seen that the prediction of sorption properties of oxyfluorinated non-woven material by quantitative analysis of the front image of the corresponding sample is to some extent possible based on data from the first octile (0..32) of the corresponding correlograms. In cases (a), (c), (e) and (g) in this range of pixel brightness, the maximum value of the correlation coefficient of the sorption capacity of experimental samples $S C$ and the values of the characteristic function $\Delta\left(S / S_{0}(B)\right)$ of the corresponding images is observed. However, since the correlation coefficient in the case of (c) takes a value $\cong 0,5$, and in the cases of (e) and $(\mathrm{g})-\cong 0,75$, the formation of the corresponding regression model is not appropriate due to the obviously poor quality of approximation of the model curve data. 

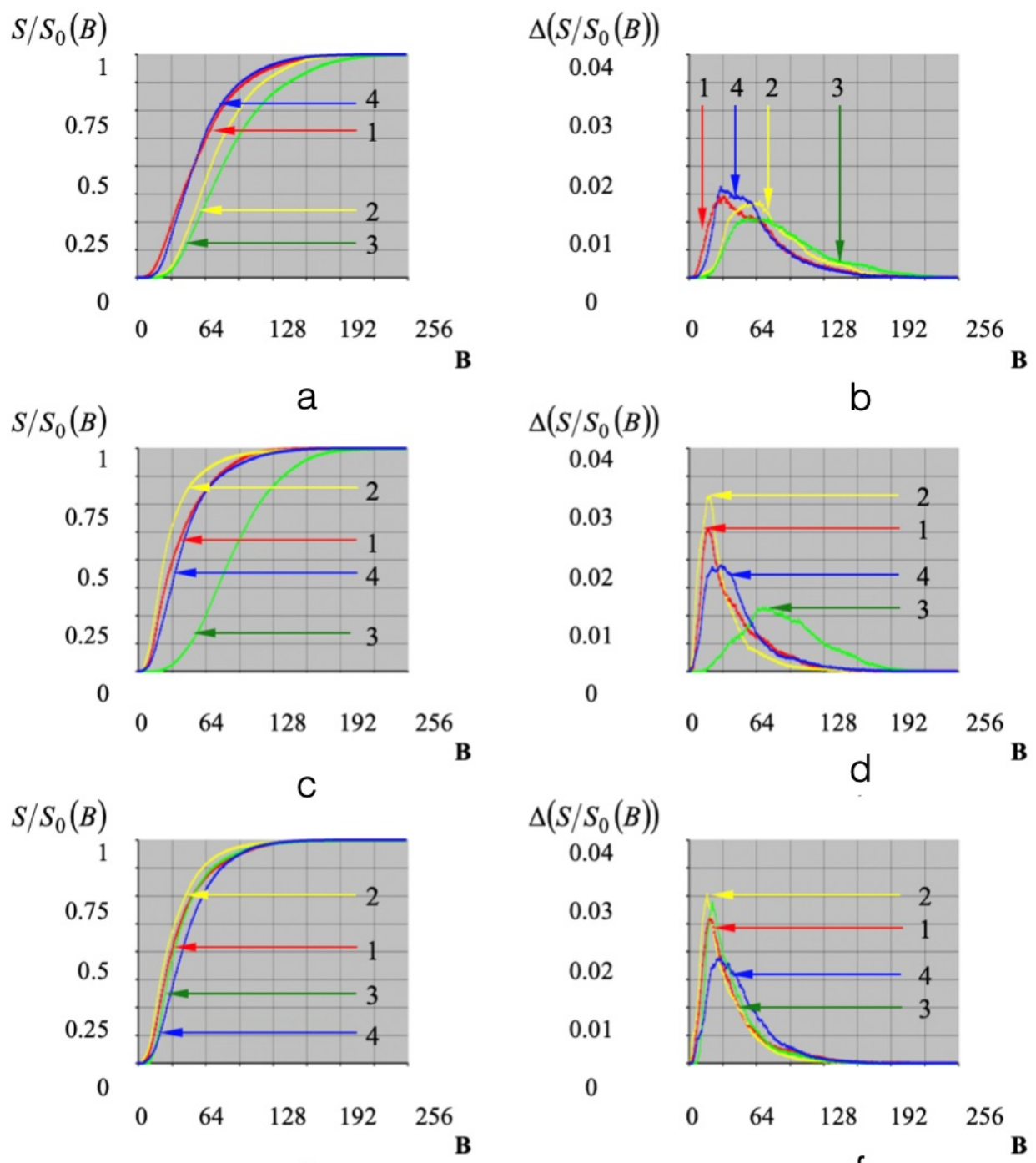

$\Delta\left(S / S_{0}(B)\right)$
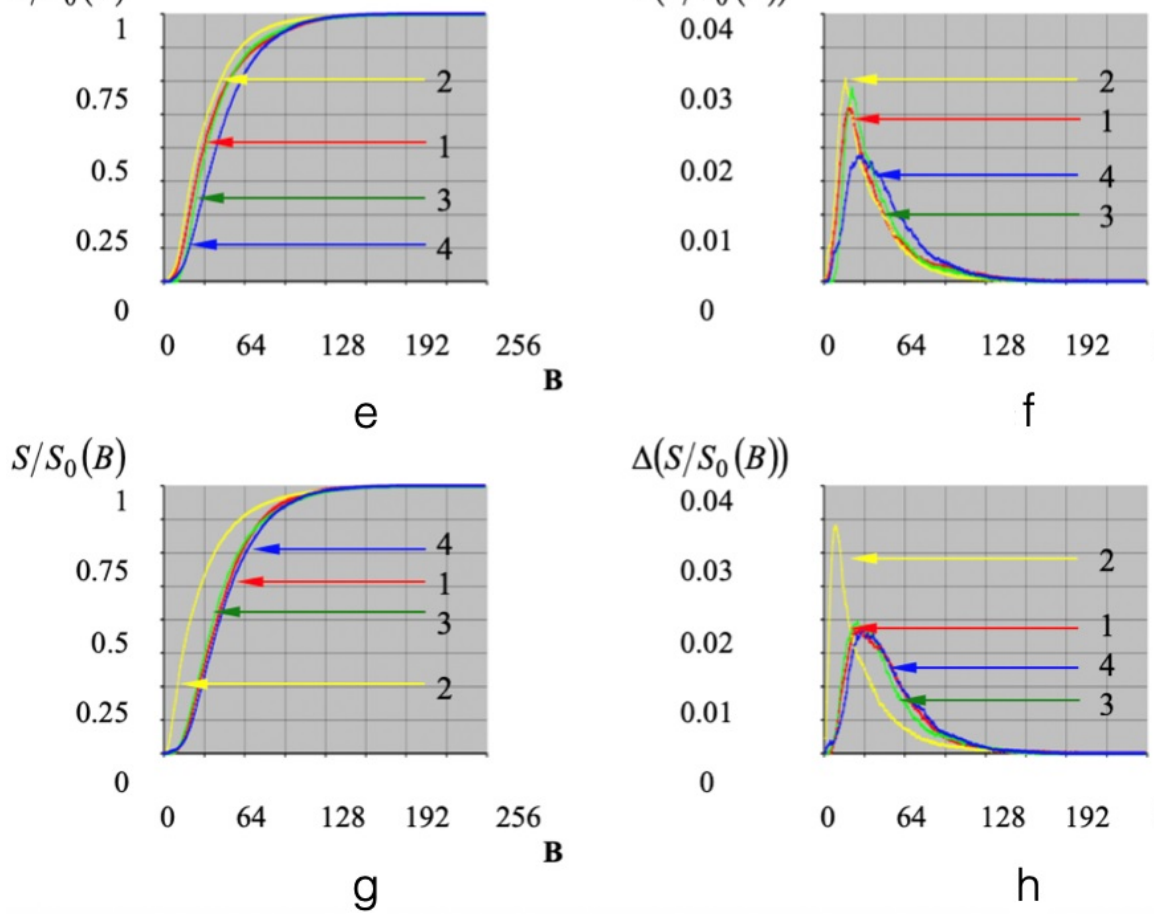

0
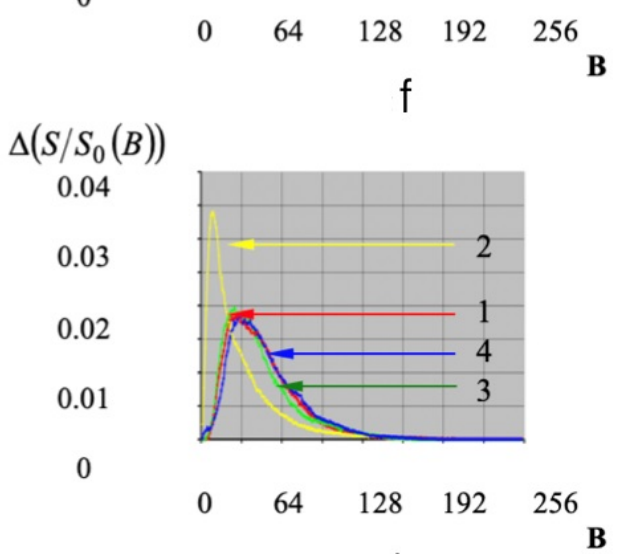

$\mathrm{h}$

FIG. 4. The characteristic functions $S / S_{0}(B)(\mathrm{a}, \mathrm{c}, \mathrm{e}, \mathrm{g})$ and $\Delta\left(S / S_{0}(B)\right)(\mathrm{b}, \mathrm{d}, \mathrm{f}, \mathrm{h})$ of the front images of the experimental samples L1(a,b), L2(c,d), L3(e,f) and L4(g,h). Curves 1-4 characterize the samples oxyfluorinated with a mixture of $7.5 \% \mathrm{~F}_{2}+10 \% \mathrm{O}_{2}+82.5 \%$ He for $0.5,1,1.5$ and 2 hours, respectively 

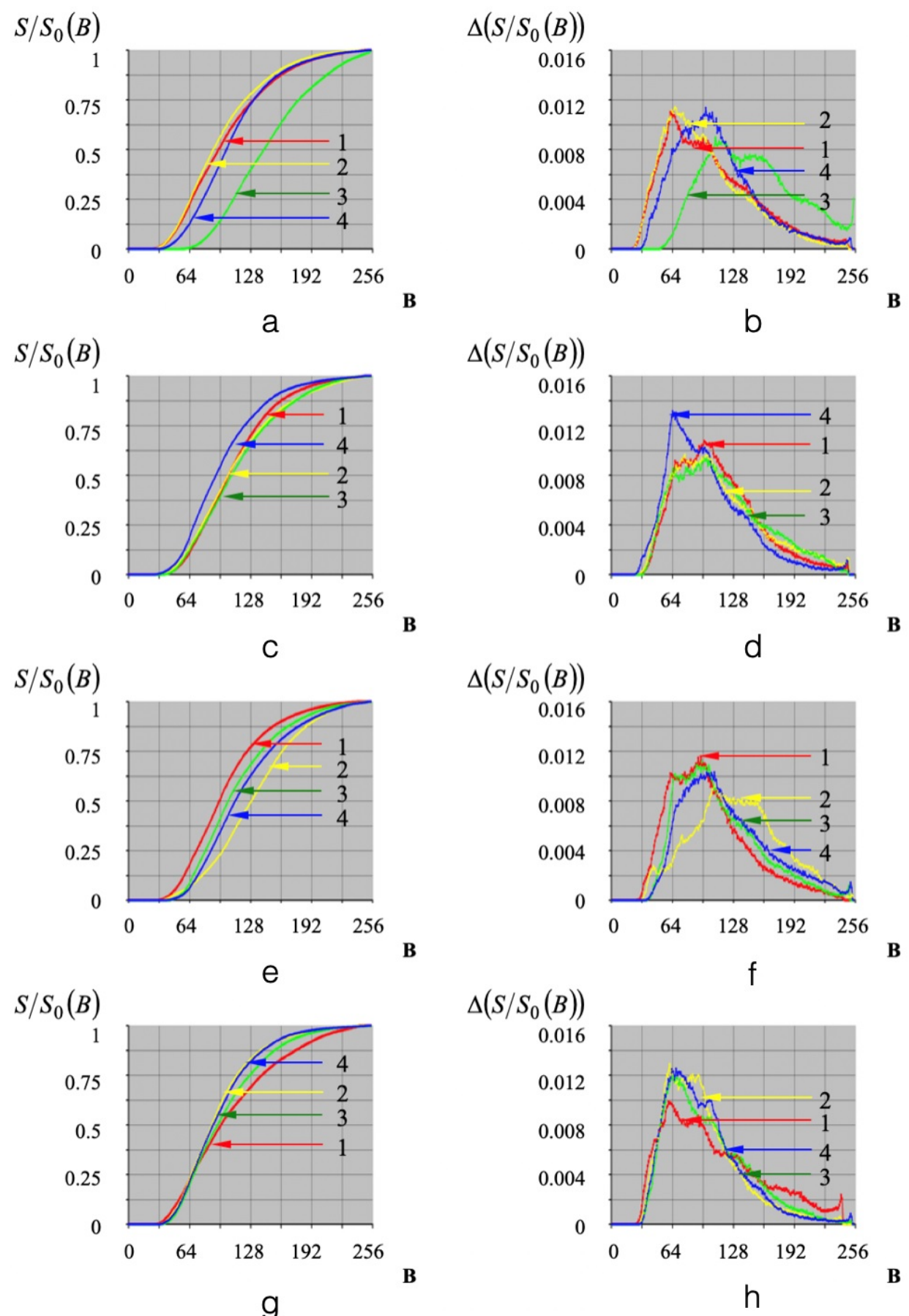

FIG. 5. The characteristic functions $S / S_{0}(B)$ (a,c,e,g) and $\Delta S / S_{0}(B)$ (b,d,f,h) of the profile images of the experimental samples L1(a,b), L2(c,d), L3(e,f) and L4(g,h). Curves 1-4 characterize samples oxyfluorinated with a mixture of $7.5 \% \mathrm{~F}_{2}+10 \% \mathrm{O}_{2}+82.5 \%$ He for $0.5,1,1.5$ and 2 hours, respectively 


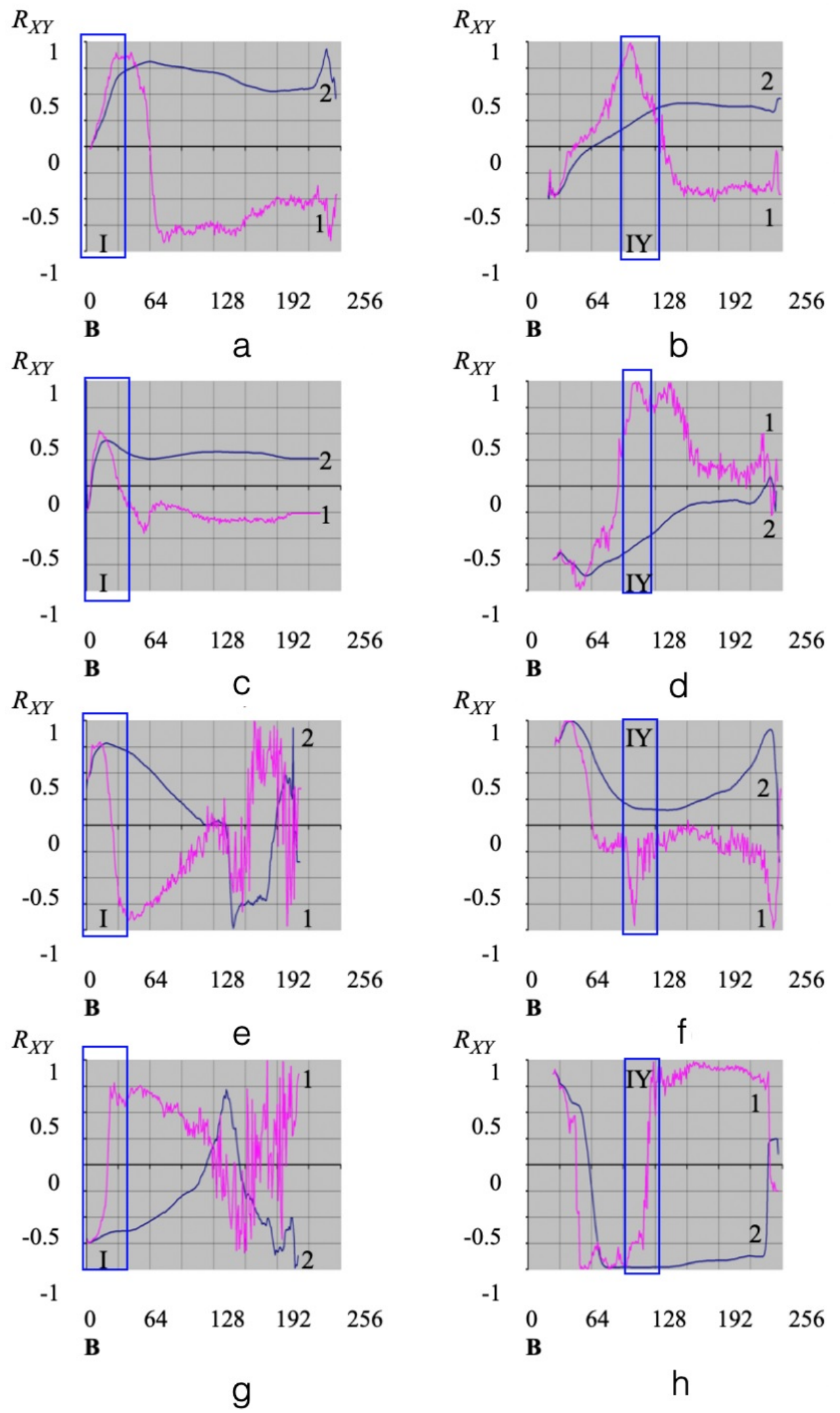

FIG. 6. The correlograms of the sorption capacity and the characteristic functions of digital planar heterogeneities for frontal $(\mathrm{a}, \mathrm{c}, \mathrm{e}, \mathrm{g})$ and profile $(\mathrm{b}, \mathrm{d}, \mathrm{f}, \mathrm{h})$ images of the experimental samples L1 $(\mathrm{a}, \mathrm{b})$, L2 (c,d), L3 (e,f) and L4 (g,h). Curves 1 and 2 correspond to the dependencies of the Pearson correlation coefficients $R_{S C ; S / S_{0}}(B)$ and $R_{S C ; \Delta\left(S / S_{0}\right)}(B)$ on the brightness threshold $B$. The first and fourth octiles are highlighted as rectangles 
In the quantitative analysis of profile sections of experimental samples, the highest modulo values of the correlation coefficients $R_{S C ; \Delta\left(S / S_{0}\right)}(B)$ observed in the first half of the fourth octiles (96..112) (highlighted by rectangles in Fig. 6(b,d,f,h) of the obtained correlograms reach values close to the maximum possible for the considered parameters

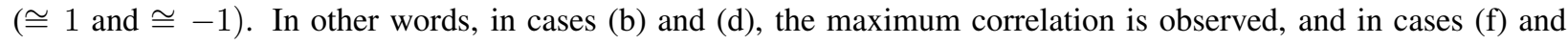
(h), the maximum anticorrelation of the characteristic function $\Delta\left(S / S_{0}(B)\right)$ and sorption capacities of experimental samples $S C$ are observed.

Thus, for statistically reliable structural and functional forecasting, it is necessary to form a quantitative assessment of sorption capacity $S C$ mainly based on the analysis of differential planar heterogeneity functions $\Delta\left(S / S_{0}(B)\right)$ for profile images of experimental samples.

Based on a set of linear regression models describing possible partial dependences of sorption capacities of experimental samples (impregnated with latex in the modes L1, L2, L3 and L4) on the corresponding values of functions $\Delta\left(S / S_{0}(B)\right)$ for all $B$ belonging to the interval $[96 ; 112]$ (the first half of the fourth octile of correlograms), four models of the form visualized in Fig. 7 and specified in Table 4 were formed:

$$
S C_{l}=\sum_{k=96}^{112} p_{k} \cdot\left(a_{k} \cdot \Delta_{l}\left(S / S_{0}\left(B_{k}\right)\right)+b_{k}\right)=\alpha_{l} \cdot \overline{\Delta_{l}\left(S / S_{0}\right)}+\beta_{l},
$$

in which $p_{k}=\frac{R_{k}^{2}}{\sum_{k=96}^{112} R_{k}^{2}} ; R_{k}^{2}=1-\frac{\sum_{l=1}^{4}\left(S C_{l}-S C_{l, k}\right)^{2}}{\sum_{l=1}^{4}\left(S C_{l}-S C_{l, k}\right)^{2}} ;\left\{S C_{l}\right\}_{4}$ - values of the sorption capacities of samples impregnated in the modes L1, L2, L3 and L4; $S C_{l, k}$ - the value of the sorption capacity of the $l$-sample calculated by the $k$-model; $\overline{S C}_{l}$ - the average value of the sorption capacity of all samples of the $l$-series; $\alpha_{l}$ and $\beta_{l}$-parameters of the $l$-functional and structural model.

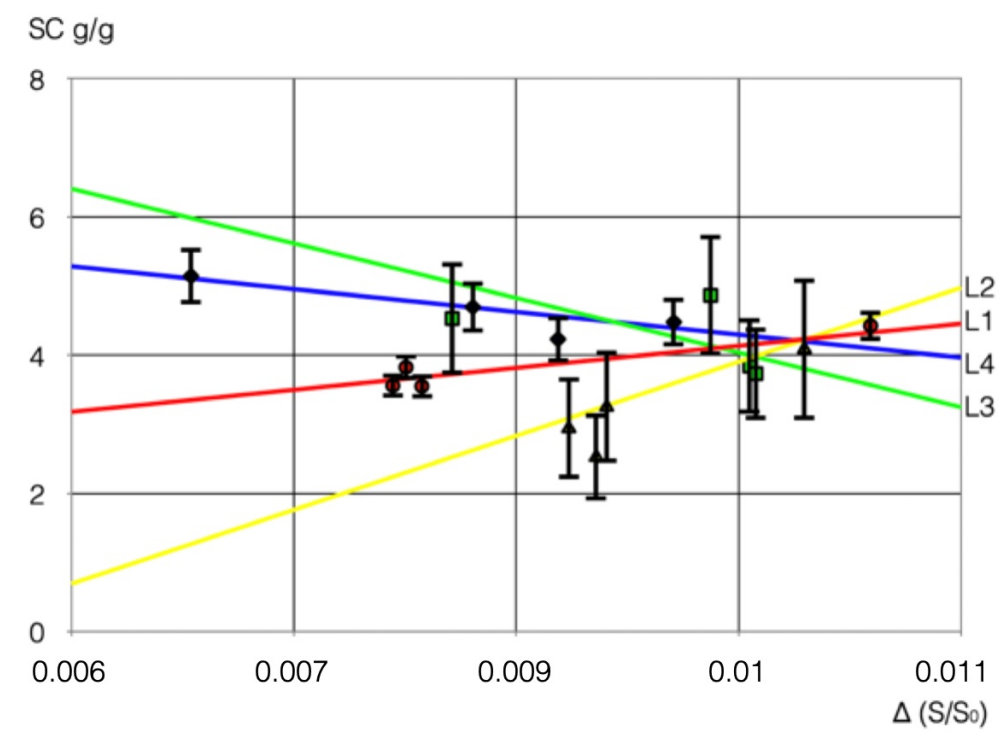

FIG. 7. The dependences of the experimental samples' sorption capacities on the values of the digital differential planar heterogeneity functions

TABLE 4. Values of parameters of functional and structural models (2) obtained by the weighted least squares (WLS) method

\begin{tabular}{|c|c|c|c|c|}
\hline Parameter & L1 & L2 & L3 & L4 \\
\hline$\alpha_{l} \pm \delta \alpha_{l}$ & $260 \pm 30$ & $860 \pm 90$ & $-630 \pm 30$ & $-260 \pm 30$ \\
\hline$\beta_{l} \pm \delta \beta_{l}$ & $1.7 \pm 0.2$ & $-4.5 \pm 0.5$ & $10.2 \pm 1$ & $6.9 \pm 0.7$ \\
\hline$R_{l}^{2}$ & $0.88 \pm 0.09$ & $0.61 \pm 0.09$ & $0.01 \pm 0,01$ & $0.73 \pm 0.07$ \\
\hline
\end{tabular}


It can be seen that the sorption capacity slightly depends on the structural characteristics of the modified nonwoven fabric at low (L1) and high (L4) concentrations of rubber in latex and strongly depends on the structural characteristics at concentrations of rubber close to a certain "threshold" value. If the proportion of rubber in the impregnating latex is less than this value, the sorption capacity increases with increasing differential planar heterogeneity. Otherwise, the larger the value $\Delta\left(S / S_{0}\right)$ becomes, the smaller the sorption capacity for latex will characterize the sample.

Thus, to ensure relatively stable values of the sorption capacity for the oxyfluorinated nonwoven fabric over its entire "effective" surface, functional covering materials similar to latexes with a percentage of rubber or less than 4 or more than $12 \%$ should be used. If it is necessary to synthesize stochastic sorption maps formed during impregnation of bicomponent polyester fiber materials, it is necessary to use mixtures similar to latex with an approximately $6 \%$ rubber content as functional ones.

The presence of the above "extreme" value is probably associated not only with the limited sedimentation stability of rubber in water, but also significantly depends on the relative position, total area, and degree of oxyfluorination of the surfaces of latex-wetted fibers.

\subsection{Multi-scale structural analysis of nonwoven fabrics}

Macroscopic optical images of the original and oxyfluorinated nonwoven fabrics fragments with dimensions of $800 \times 1000$ microns are shown in Fig. 8 .
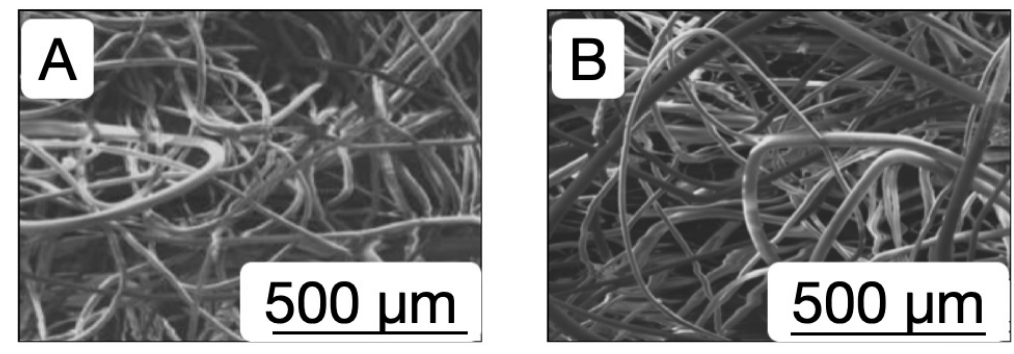

FIG. 8. SEM-images of nonwoven fabrics surfaces made of polyester fibers based on PET initial (A) and oxyfluorinated (B) with a gas mixture of $7.5 \% \mathrm{~F}_{2}+10 \% \mathrm{O}_{2}+82.5 \% \mathrm{He}$

Neither visual nor quantitative analysis of these fibrous materials images made it possible to statistically reliably establish the presence and degree of the oxyfluorination influence on the material macroscopic structure.

When analyzing SEM-images (with dimensions of $250 \times 350$ microns) of the same samples' fragments at the microscopic level (at the lowest magnification available for the Jeol7500F microscope (Japan)) differences in the observed textural heterogeneity degrees of the initial and oxyfluorinated fibers surfaces become noticeable (Fig. 9), but a quantitative description of the corresponding nanoscale inhomogeneities is still impossible (due to the fact that at a low resolution of the electron microscope, the characteristic size for one pixel of the generated image exceeds 1 micron).
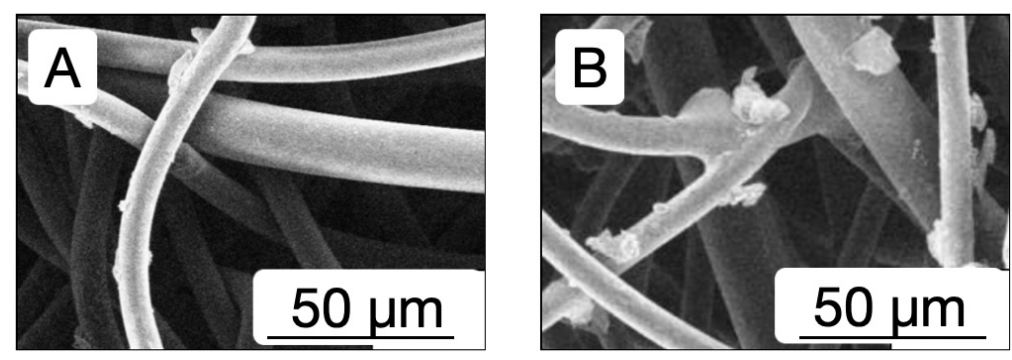

FIG. 9. SEM-images of polymer fiber groups for initial (A) and oxyfluorinated (B) with a gas mixture of $7.5 \% \mathrm{~F}_{2}+10 \% \mathrm{O}_{2}+82.5 \% \mathrm{He}$

Finally, at the maximum magnification for the Jeol7500F (Fig. 10), it is possible to fix and then model the nanotextures of the original and oxyfluorinated nonwoven fibers using the original method of the quantitative SEM-images analysis developed earlier [19-21]. The characteristic size for one pixel in this case is $\sim 1 \mathrm{~nm}$. 

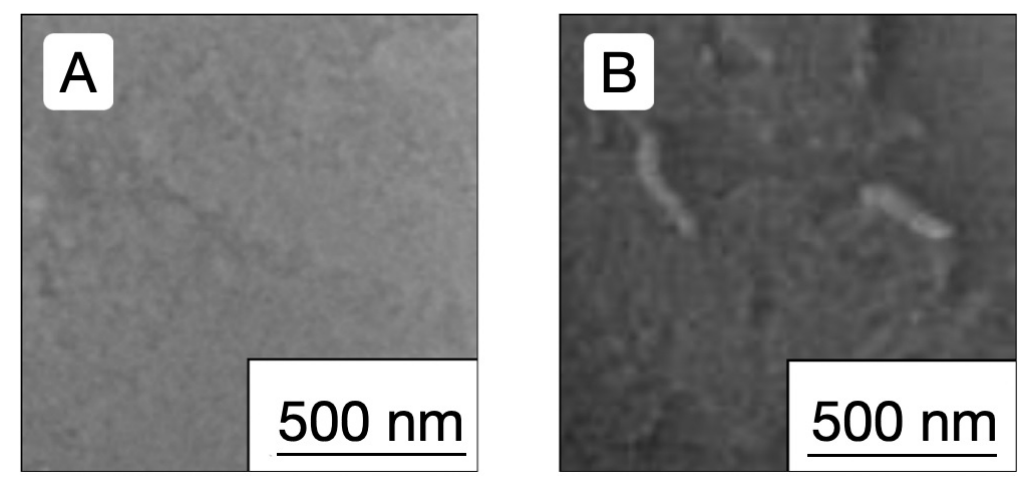

FIG. 10. SEM-images of polymer fiber surfaces: initial (A) and oxyfluorinated (B) with a gas mixture of $7.5 \% \mathrm{~F}_{2}+10 \% \mathrm{O}_{2}+82.5 \% \mathrm{He}$

\subsection{Dependence of the fiber surfaces' nanotexture on the duration of modification}

SEM-images (with dimensions of $1.2 \times 1.2$ microns) of the surfaces' fragments for the original and treated with a gas mixture of $7.5 \% \mathrm{~F}_{2}+10 \% \mathrm{O}_{2}+82.5 \%$ He fibers (with modification durations of $0.5,1,1.5$ and 2 hours) are shown in Fig. 11.
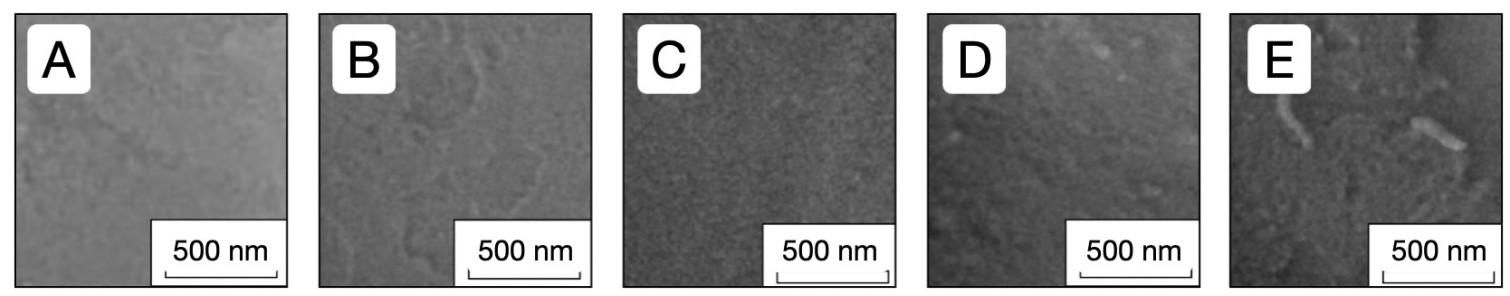

FIG. 11. SEM-images of the PET fiber surfaces: initial (A) and oxyfluorinated with a gas mixture of $7.5 \% \mathrm{~F}_{2}+10 \% \mathrm{O}_{2}+82.5 \% \mathrm{He}$ for $0.5(\mathrm{~B}), 1(\mathrm{C}), 1.5(\mathrm{D})$, and $2(\mathrm{E})$ hours

The study of the nanostructural transformations dynamics of fiber surfaces showed that the localization region of the morphological spectrum (Fig. 12), which characterizes the sample's SEM-image, increases with increasing the oxyfluorination duration, probably reaches the maximum value, and then decreases. (Fig. 13). The dynamics of the morphological spectrum expressed in dimensionless units of the radius of the localization region and the derivative of the model (1) are anticorrelated at the level of $0.78 \pm 0.08$.
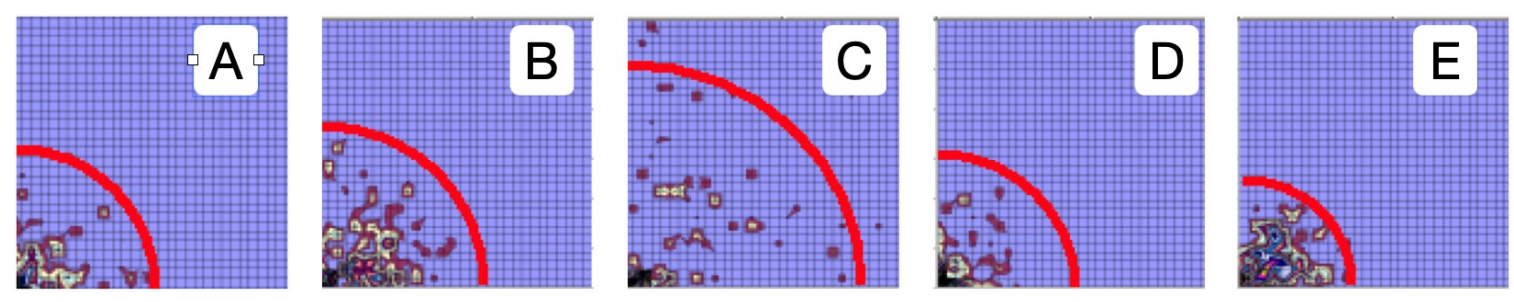

FIG. 12. Morphological spectra of the surfaces SEM-images for material-forming PET fibers: initial (A) and oxyfluorinated with a gas mixture of $7.5 \% \mathrm{~F}_{2}+10 \% \mathrm{O}_{2}+82.5 \% \mathrm{He}$ for $0.5(\mathrm{~B}), 1$ (C), 1.5 (D) and 2 (E) hours. The line indicates the radius of localization of the morphological spectrum

Since it is known [22] that the PET surface hydrophilicity increases sharply during gas-phase treatment with mixtures whose active reagents are fluorine and oxygen, when latex hits the effective surface of a nonwoven fabric, the processes of diffusion of latex as a colloidal solution into the thickness of the nonwoven material and intensive water sorption by the PET fibers surface, hydrophilized by oxyfluorination begin to compete. This phenomenon increases the uncertainty in predicting the sorption capacity at close to critical values of the rubber concentration in latex. 


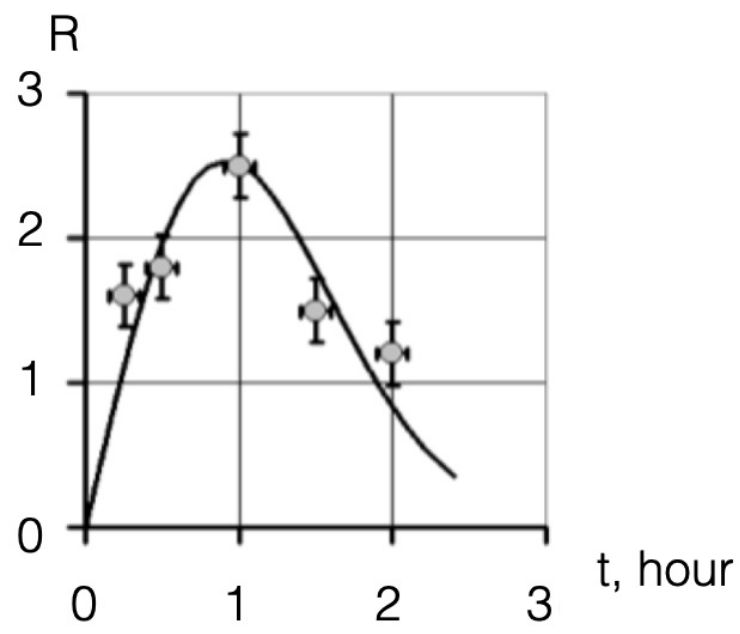

FIG. 13. Dependence of the morphological spectrum localization radius of the polymer fiber surface SEM-image on the duration of its oxyfluorination

However, the relationship between the latex-sorption capacity increment of the material, and the values of the regions radii for the morphological spectra localization, which characterize the nanoscale SEM-images of fibers surfaces, describes the mechanism by which changes in setting of the modification mode (the duration of the gas-phase oxyfluorination) manages the functional material properties (e.g., sorption capacity).

\section{Conclusion}

An approach to structural and functional modeling of composite materials based on oxyfluorinated nonwovens has been developed. The structural component of the model can be represented in the form of integral or differential characteristic functions of planar heterogeneity $S / S_{0}(B)$ and $\Delta S / S_{0}(B)$ and in the form of expansion into a twodimensional Fourier series with a morphological spectrum $\left\{A_{k l}\right\}_{N \times N}$.

The presence of correlations between the sorption capacities (for latex) and the structural characteristics of the optical image of experimental samples was established using classical methods of correlation analysis of data. The obtained functional and structural model allows us to quantify the expected values of the sorption capacity of bicomponent polyester fiber materials under certain restrictions on the chemical composition of the impregnating liquid-phase mixtures. It was found that the uncertainty in the predicted values of the sorption capacity increases sharply with the $\cong 6 \%$ content of rubber in latex, which indicates the determining influence of the particular structure of a particular sample on its sorption properties relative to the above-mentioned impregnating mixture. Since it is impossible to form structurally identical non-woven fabrics (due to the peculiarities of manufacturing technology), it is recommended to use compositions similar to latex with a far from extreme percentage of rubber $(<4$ or $>12 \%)$ when finishing oxyfluorinated fiber materials with covering compounds.

\section{Acknowledgments}

This work was funded by Ministry of Science and Higher Education of the Russian Federation (State assignment "Structure and properties of the polymer materials produced using system of chemically, thermally and / or mechanically induced both surface and bulk modification techniques", topic number FZRR-2020-0024, code 06992020-0024).

\section{References}

[1] Li Y., Ma T., Yang S.T., Kniss D.A. Thermal compression and characterization of three-dimensional nonwoven PET matrices as tissue engineering scaffolds. Biomaterials, 2001, 22(6), P. 609-618.

[2] Hyde G.K., Scarel G., Peng Q., Lee K., Gong B., Roberts K.G., Roth K.M., Hanson C.A., Devine C.K., Stewart S.M., Hojo D., Na J.-S., Jur J.S., Parsons G.N., Spagnola J.C. Atomic layer deposition and abrupt wetting transitions on nonwoven polypropylene and woven cotton fabrics. Langmuir: the ACS journal of surfaces and colloids, 2010, 26(4), P. 2550-2558.

[3] Du D., Li P., Ouyang J. Graphene coated nonwoven fabrics as wearable sensors. Journal of Materials Chemistry C, 2016, 4(15), P. 3224-3230.

[4] Smirnov A.V., Genis A.V. Effect of process parameters on the structure and physicomechanical properties of nonwoven materials manufactured by aerodynamic spinning from polymer solution. Fibre Chemistry, 2002, 34(6), P. 400-406.

[5] Kumar A., Gaurav, Malik A.K., Tewary D.K., Singh B. A review on development of solid phase microextraction fibers by sol-gel methods and their applications. Analytica Chimica Acta, 2008, 610(1), P. 1-14. 
[6] Bolbasov E.N., Stankevich K.S., Sudarev E.A., Kudryavtseva V.L., Tverdokhlebov S.I., Bouznik V.M., Antonova L.V., Matveeva V.G., Anissimov Y.G. The investigation of the production method influence on the structure and properties of the ferroelectric nonwoven materials based on vinylidene fluoride - tetrafluoroethylene copolymer. Materials Chemistry and Physics, 2016, 182, P. 338-346.

[7] Moheman A., Alam M.S., Mohammad A. Recent trends in electrospinning of polymer nanofibers and their applications in ultra thin layer chromatography. Advances in Colloid and Interface Science, 2016, 229, P. 1-24.

[8] Bao L., Wang Y., Wakatsuki K., Morikawa H., Baba T., Fukuda Y. Development of a high-density nonwoven structure to improve the stab resistance of protective clothing material. Industrial Health, 2017, 55(6), P. 513-520.

[9] Lipinski C.A., Lombardo F., Dominy B.W., Feeney P.J. Experimental and computational approaches to estimate solubility and permeability in drug discovery and development settings. Advanced Drug Delivery Reviews, 1997, 23(1-3), P. 3-25.

[10] Ng E.P., Mintova S. Nanoporous materials with enhanced hydrophilicity and high water sorption capacity. Microporous and Mesoporous Materials, 2008, 114(1-3), P. 1-26.

[11] Liu K., Jiang L., Yao X. Recent developments in bio-inspired special wettability. Chemical Society Reviews, $2010,39(8)$, P. $3240-3255$.

[12] Hes L., de Araujo M., Djulay V.V. Effect of mutual bonding of textile layers on thermal insulation and thermal contact properties of fabric assemblies. Textile Research Journal, 1996, 66(4), P. 245-250.

[13] Tovmash A.V., Polevov V.N., Mamagulashvili V.G., Chernyaeva G.A., Shepelev A.D. Fabrication of sorption-filtering nonwoven material from ultrafine polyvinyl alcohol carbonized fibres by electrospinning. Fibre Chemistry, 2005, 37(3), P. 187-191.

[14] Radetic M.M., Jocic D.M., Jovancic P.M., Petrovic Z.Lj., Thomas H.F. Recycled wool-based nonwoven material as an oil sorbent. Environmental Science and Technology, 2003, 37(5), P. 1008.

[15] Prorokova N.P., Istratkin V.A., Kumeeva T.Y., Vavilova S.Y., Kharitonov A.P., Bouznik V.M. Improvement of polypropylene nonwoven fabric antibacterial properties by the direct fluorination. RSC Advances, 2015, 5(55), P. 44545-44549.

[16] Bondar Y.V., Kim H.J., Lim Y.J. Sulfonation of (glycidyl methacrylate) chains grafted onto nonwoven polypropylene fabric. Journal of Applied Polymer Science, 2007, 104(5), P. 3256-3260.

[17] Poncin-Epaillard F., Brosse J.C., Falher T. Cold plasma treatment: surface or bulk modification of polymer films. Macromolecules, 1997, 30(15), P. 4415-4420.

[18] Dedov A.V., Nazarov V.G. Processed nonwoven needlepunched materials with increased strength. Fibre Chemistry, 2015, 47(2), P. 121-125.

[19] Isaev E.A., Pervukhin D.V., Kornilov V.V., Tarasov P.A., Grigoriev A.A., Rudyak Yu.V., Rytikov G.O., Nazarov V.G. Platelet adhesion quantification to fluorinated polyethylene from the structural characteristics of its surface. Mathematical biology and bioinformatics, 2019, 14(2), P. 420-429.

[20] Drozdov S.A., Nazarov V.G., Nozdrachev S.A., Rudyak Yu.V., Rytikov G.O. The polymer composites morphological structure simulation. Nanosystems: Physics, Chemistry, Mathematics, 2017, 8(1), P. 137-145.

[21] Petrushin V.N., Rudyak Y.V., Rytikov G.O. The holistic method of the surface structure characterization. Proceedings of "2016 14th International Baltic Conference on Atomic Layer Deposition (BALD 2016)". St. Petersburg, Russia, 2-4 October 2016, P. 15-19.

[22] Nazarov V.G., Doronin F.A., Evdokimov A.G., Rytikov G.O., Stolyarov V.P. Oxyfluorination-Controlled Variations in the Wettability of Polymer Film Surfaces. Colloid Journal, 2019, 81, P. 146-157. 